Pacific Journal of Mathematic 


\title{
IDEAL STRUCTURE IN GENERALIZED GROUP ALGEBRAS
}

\author{
KJELD B. LAURSEN
}

We study the algebra $L^{1}(G, A)$ of Bochner-integrable functions from a locally compact topological group $G$ to a Banach algebra $A$. First we characterize closed ideals in $L^{1}(G, A)$ as subspaces that are translation invariant in a certain sense (Theorem 2.2). After that we establish some generalizations of Wiener's tauberian theorem. The class of algebras under consideration consists of strongly semi-simple and completely regular Banach algebras. After this, in §3, we deal with spectral synthesis. Our main result (Corollary 3.6) states that if $A$ does not admit spectral synthesis then neither does $L^{1}(G, A)$. In $\$ 4$ we apply the theory of completely regular, strongly semi-simple Banach algebras to obtain some conditions sufficient to ensure that a given ideal is the intersection of the maximal regular ideals containing it.

An introduction to the theory of tensor products of vector spaces can be found in several places, for instance in [16] or [5]. The question of norming a tensor product is treated in the references mentioned; the greatest cross norm $\gamma$ is defined there. We include a definition for completeness.

Definition 1.1. Let $E_{1}, E_{2}$ be normed vector spaces and suppose $t \in E_{1} \otimes E_{2}$. We define the greatest cross norm $\gamma$ by

$$
\gamma(t)=\inf \sum\left|x_{i}\right|\left|y_{i}\right|
$$

with inf taken over all representatives $\sum x_{i} \otimes y_{i}=t$. The completion of $E_{1} \otimes E_{2}$ with respect to $\gamma$ is denoted by $E_{1} \otimes_{r} E_{2}$.

The following structure theorems will be used extensively in this paper, sometimes without explicit mention. Proposition 1.4 provides one of the major justifications for the study of vector valued group algebras.

Proposition 1.2 [5]. Let $G$ be a locally compact group and $A$ a Banach algebra. Then

$$
L^{1}(G, A)=L^{1}(G) \otimes_{\gamma} A
$$

where $=$ denotes an isometric isomorphism.

REMARK 1.3. In [5, p. 59] proposition 1.2 is proved when $A$ is 
a vector space. However, it is not difficult to extend the validity of the result to algebras.

Proposition 1.4. Let $G$ and $H$ be locally compact groups. Then

$$
L^{1}(G \times H)=L^{1}(G) \otimes_{\gamma} L^{1}(H) .
$$

Proposition 1.4 is a special case of Proposition 1.2 via the identification $L^{1}(G \times H)=L^{1}\left(G, L^{1}(H)\right)$ (Cf. [20]).

2. Ideal structure. Tauberian theorems. If $G$ is a locally compact group the following theorem about closed one-sided ideals in $L^{1}(G)$ holds (Cf. e.g., [14, p. 374]) :

A closed subspace $I$ of $L^{1}(G)$ is a left (right) ideal if and only if it is invariant under left (right) translations, i.e., if and only if $x(\cdot) \in I \Rightarrow x\left(g_{0} \cdot\right) \in I\left(x\left(\cdot g_{0}\right) \in I\right)$ for every $g_{0} \in G$.

Grove [6] has shown that this assertion is not valid in a generalized group algebra. Here we show that if we consider a different concept of translation the theorem is true. We make the following definition.

Definition 2.1. We say that a subspace $I$ of $L^{1}(G, A)(G$ is a locally compact group, $A$ a Banach algebra) is left $A$-translation invariant if

(i) $x(\cdot) \in I \Rightarrow x\left(g_{0} \cdot\right) \in I \quad$ for every $g_{0} \in G$

(ii) $x(\cdot) \in I \Rightarrow a x(\cdot) \in I \quad$ for every $a \in A$.

Analogously, we define right $A$-translation invariance by replacing (i) by

(i') $x(\cdot) \in I \Rightarrow x\left(\cdot g_{0}\right) \in I \quad$ for every $g_{0} \in G$ and (ii) by

(ii') $x(\cdot) \in I \Rightarrow x(\cdot) a \in I \quad$ for every $a \in A$.

We can then prove the following

THEOREM 2.2. Let $G$ be a locally compact group and $A$ a Banach algebra with approximate identity. A closed subspace $I$ of $L^{1}(G, A)$ is a left (right, 2-sided) ideal if and only if I is left (right, left and right) A-translation invariant.

Proof. We prove this for a left ideal. So let $I$ be a closed left ideal. Using the notation $x_{g}$ for the function $x(g \cdot)$ and letting $\left\{u^{\alpha}\right\}$ be an approximate identity for $L^{1}(G, A)$ we have (as in the numerical case) that 


$$
\begin{aligned}
\left(u_{g_{0}-1 * x}^{\alpha}\right)(g) & =\int_{G} u^{\alpha}\left(g_{0}^{-1} g^{\prime}\right) x\left(g^{\prime-1} g\right) d g^{\prime} \\
& =\int_{G} u^{\alpha}\left(g^{\prime}\right) x\left(g^{\prime-1} g_{0} g\right) d g^{\prime} \\
& =\left(u^{\alpha} * x\right)_{g_{0}}(g),
\end{aligned}
$$

so that for $x \in I$

$$
x_{g_{0}}=\lim \left(u^{\alpha} * x\right)_{g_{0}}=\lim \left(g_{g_{0}}^{\alpha} * x\right) \in I .
$$

This shows that $x \in I \Rightarrow x_{g} \in I$ for every $g \in G$. The proof of (ii), Definition 2.1 is as follows

$$
\begin{aligned}
x \in I, a \in A \Rightarrow & a u^{\alpha} * x \in I \\
& \lim a u^{\alpha} * x \in I \\
& a \lim u^{\alpha} * x=a x \in I .
\end{aligned}
$$

Thus we have shown that a closed left ideal in $L^{1}(G, A)$ is left $A$-translation invarient.

The proof of the converse implication is based on approximation by means of a sort of Riemann sums.

Let $I$ be a closed subspace of $L^{1}(G, A)$ such that $I$ is left $A$ translation invariant. We must show that $x \in I, y \in L^{1}(G, A) \Rightarrow y * x \in I$. Since $I$ is closed and continuous functions with compact support are dense in $L^{1}(G, A)$ it suffices to show that $x \in I \Rightarrow y * x \in I$ for any continuous $y \in L^{1}(G, A)$ with compact support. So let $y$ be such a function with support $K$. From what is given about $I$ it follows that

$$
\alpha y\left(g_{0}\right) x_{g_{0}} \in I \quad \text { for any } g_{0} \in G \text { and } \alpha \in C .
$$

But

$$
y * x=\int_{G} y\left(g^{\prime}\right) x_{g^{\prime}-1} d g^{\prime}=\int_{K} y\left(g^{\prime}\right) x_{g^{\prime}}-1 d g^{\prime}
$$

and the last term can be approximated arbitrarily well by sums of the form

$$
\sum_{k} y\left(g_{k}\right) x_{g_{k}} \cdot
$$

This can be seen in the following way: as in the numerial case we can show that $x_{g}$ is a continuous function of $g$ with respect to the norm in $L^{1}(G, A)$. Since $y$ is continuous it follows easily that $y(g) x_{g}$ is continuous (here we use pointwise multiplication). Moreover, $y(g) x_{q}=0$ outside $K$. From the compactness of $K$ and the uniform continuity of $y(g) x_{g}$ on $K$ it follows that corresponding to a given $\varepsilon>0$ there are finitely many measurable disjoint sets $K_{i}(i=1,2, \cdots, n)$ such that $g^{\prime}, g^{\prime \prime} \in K_{i} \Rightarrow\left|y\left(g^{\prime}\right) x_{g^{\prime}}-y\left(g^{\prime \prime}\right) x_{g^{\prime \prime}}\right|<\varepsilon$. If we choose $g_{i} \in K_{i}$, and define 


$$
\varphi(g)= \begin{cases}\sum_{i=1}^{n} 1_{K_{i}}(g) y\left(g_{i}\right) x_{g_{i}}(g) & g \in K \\ 0 & g \notin K\end{cases}
$$

where $1_{K_{i}}$ is the characteristic function of $K_{i}(i=1, \cdots, n)$ then $\varphi$ is clearly integrable; moreover, an application of Fubini's theorem yields that

$$
\begin{aligned}
& \int_{G}\left|\int_{K} y\left(g^{\prime}\right) x_{g^{\prime}-1}(g) d g^{\prime}-\sum_{k} y\left(g_{k}\right) x_{g_{\bar{k}}^{-1}}(g) \mu\left(K_{k}\right)\right| d g \\
& \quad=\int_{G}\left|\sum_{k} \int_{K}\right| y\left(g^{\prime}\right) x_{g^{\prime}-1}(g)-y\left(g_{k}\right) x_{g_{\bar{k}^{-1}}}(g)\left|d g^{\prime}\right| d g \\
& \quad \leqq \sum_{k} \int_{G} \int_{K_{k}}\left|y\left(g^{\prime}\right) x_{g^{\prime}-1}(g)-y\left(g_{k}\right) x_{g_{l_{k}}}(g)\right| d g^{\prime} d g \\
& \quad=\sum_{k} \int_{K_{k}} \int_{G}\left|y\left(g^{\prime}\right) x_{g^{\prime}-1}(g)-y\left(g_{k}\right) x_{g_{\bar{k}}}(g)\right| d g d g^{\prime} \\
& \quad<\sum_{k} \int_{K_{k}} \varepsilon d g^{\prime}=\varepsilon \sum_{k} \int_{K_{k}} d g^{\prime}=\varepsilon \mu(K) .
\end{aligned}
$$

Since $I$ is closed this shows that $y * x \in I$, i.e., $I$ is a left ideal. This proves the theorem.

From the proof of Theorem 2.2 we get

Corollary 2.3. If $x_{1}, x_{2} \in L^{1}(G, A)$ and $\varepsilon>0$ is given then there is a finite sum $h$ of A-translations of $x_{1}$ such that $\left|x_{1} * x_{2}-h\right|<\varepsilon$.

Later, when we get to the spectral synthesis we shall make use of Theorem 2.2. However, as a kind of introduction to the spectral synthesis we shall concern ourselves with a special case, namely various formulations of Wiener's tauberian theorems and the extensions of these to generalized group algebras. It is well known that an important step in one standard proof of the so-called generalized Wiener's tauberian theorem is a proof of the fact that if $G$ is a locally compact abelian group then every closed proper ideal in $L^{1}(G)$ is contained in a maximal regular ideal. A necessary condition that $L^{1}(G, A)$ have this property is that the range algebra $A$ have it (see Corollary 3.5).

Therefore, we are led to the theory of the so-called tauberian algebras which we define as follows (cf. [21]).

Definition 2.4. A Banach algebra $A$ is tauberian if every closed proper 2-sided ideal has a nonempty hull, i.e., if every proper closed 2 -sided ideal is contained in a maximal regular 2-sided ideal.

Apparently the theory of these algebras is not yet very extensive; 
the results that are of interest in this context make one or two other assumptions about the algebras in question. Consequently, we start out by considering these concepts, i.e., that of strong semi-simplicity and that of complete regularity.

Definition 2.5. Let $A$ be an algebra. The strong structure space $R S(A)$ is the collection of all maximal regular 2-sided ideals in $A$ with the hull-kernel topology. The strong radical, $R_{s}(A)$ is defined by

$$
R_{s}(A)=\bigcap_{M \in R S(A)} M .
$$

If $R_{s}(A)=\{0\}, A$ is strongly semi-simple.

Let $B_{1}$ and $B_{2}$ be Banach-algebras, $B_{3}=B_{1} \otimes_{\gamma} B_{2}$. Following [4] we define

$$
T: J_{1} \times J_{2} \longrightarrow J_{3}
$$

where $J_{i}$ is the collection of closed 2 -sided modular ideals in $B_{i}(i=1,2,3)$ and

$$
\widetilde{T}: R S\left(B_{1}\right) \times R S\left(B_{2}\right) \longrightarrow R S\left(B_{1} \otimes_{\gamma} B_{2}\right) .
$$

Let $\left(I_{1}, I_{2}\right) \in J_{1} \times J_{2}$ and define

$$
R: B_{1} \otimes_{r} B_{2} \longrightarrow B_{1} / I_{1} \otimes_{r} B_{2} / I_{2}
$$

by

$$
t=\sum x_{i} \otimes y_{i} \in B_{1} \otimes{ }_{\gamma} B_{2} \Longrightarrow R(t)=\sum x_{i} / I_{1} \otimes y_{i} / I_{2} \in B_{1} / I_{1} \otimes_{\gamma} B_{2} / I_{2} .
$$

Then

$$
T\left(I_{1}, I_{2}\right) \equiv \operatorname{kernel}(R) \text {. }
$$

It is not difficult to see that $T\left(I_{1}, I_{2}\right) \in J_{3}$. If

$$
\left(M_{1}, M_{2}\right) \in R S\left(B_{1}\right) \times R S\left(B_{2}\right) \subset J_{1} \times J_{2}
$$

then let $M_{3} \in R S\left(B_{1} \otimes_{\gamma} B_{2}\right)$ be a maximal modular ideal containing $T\left(M_{1}, M_{2}\right)$ and define

$$
\widetilde{T}\left(M_{1}, M_{2}\right)=M_{3} \text {. }
$$

$T$ and $\widetilde{T}$ are defined in [4] without use of the blanket assumption of that paper that $B_{1}$ and $B_{2}$ have identities. Clearly, therefore, Lemma 2 [4] holds; moreover Theorem 1 [4] shows that $\widetilde{T}$ is closed when the hull-kernel topology is used. If $B_{1}$ and $B_{2}$ have identities $\widetilde{T}$ is injective [4]; if $B_{1}$ or $B_{2}$ is commutative $\widetilde{T}$ is injective and sur- 
jective even when no identities are present [12]. It is not known to the author whether $\widetilde{T}$ has these two properties in general. Generally, we shall assume $\widetilde{T}$ to have these properties, i.e., to be bijective.

About strongly semi-simple generalized group algebras we have the following immediate generalization of [12, Corollary 1]:

THEOREM 2.6. Let $G$ be a locally compact group, $A$ a Banach algebra. Assume $\widetilde{T}: R S\left(L^{1}(G)\right) \times R S(A) \rightarrow R S\left(L^{1}(G, A)\right)$ to be bijective. Then $L^{1}(G, A)$ is strongly semi-simple if and only if $L^{1}(G)$ and $A$ are strongly semi-simple.

Next, we turn to the completely regular algebras. Following [21] we have

Definition 2.7. A Banach algebra $A$ is completely regular if $R S(A)$ satisfies these conditions:

(i) $R S(A)$ is Hausdorff ;

(ii) For every $M \in R S(A)$ there is an open set 0 such that $M \in 0 \subset R S(A)$ and such that $k(0)$ is a regular ideal (here $k$ denotes kernel).

In $[21, \mathrm{p} .178]$ it is shown that the above conditions are equivalent to the single condition that $R S(\widetilde{A})$ be Hausdorff, where $\widetilde{A}$ is the smallest Banach algebra with identity containing $A$.

We first prove

THEOREM 2.8. If $B_{1}$ and $B_{2}$ are completely regular Banach algebras and if $\widetilde{T}$ is injective then

$$
\widetilde{T}: R S\left(B_{1}\right) \times R S\left(B_{2}\right) \longrightarrow R S\left(B_{1} \otimes_{\gamma} B_{2}\right)
$$

is a homeomorphism so that if $\widetilde{T}$ is bijective then $B_{1} \otimes_{r} B_{2}$ is completely regular.

Proof. Let $O_{i}$ be an open set in $R S\left(B_{i}\right)$ for which $k\left(O_{i}\right)=I_{i}$ is a regular ideal, $i=1,2$. Such sets exist in abundance by Definition 2.7 ii). The closure of $O_{i}=h\left(k\left(O_{i}\right)\right)=h\left(I_{i}\right)$ is a compact set [15, Th. 2.6.4]. Let $0=\widetilde{T}\left(0_{1} \times 0_{2}\right)$. We first show that 0 has compact closure. Again using [15, Th. 2.6.4] this will be accomplished by showing that $k(0)$ is a regular ideal. We use the mapping $T$ and Lemma 2 of [4]: $T\left(I_{1}, I_{2}\right)$ is a regular ideal and if we can show that $T\left(I_{1}, I_{2}\right) \subset k(0)$ then $k(0)$ is regular. But 


$$
\begin{aligned}
T\left(I_{1}, I_{2}\right) & \cong \bigcap_{\substack{M i \in O_{i} \\
i=1,2}} T\left(M_{1}, M_{2}\right) \\
& \cong \bigcap_{\substack{M_{2} \in O_{2} \\
i=1,2}} T\left(M_{1}, M_{2}\right) \\
& =\bigcap_{M \in 0} M=k(0) .
\end{aligned}
$$

Therefore $h(k(0))$ is a compact set. Now, since $B_{1}, B_{2}$ are completely regular $h\left(k\left(0_{1}\right)\right) \times h\left(k\left(0_{2}\right)\right)$ is Hausdorff. $\widetilde{T}\left(h\left(k\left(0_{1}\right) \times\left(h\left(k\left(0_{2}\right)\right)\right)\right)\right.$ is closed in the relative topology of $\widetilde{T}\left(R S\left(B_{1}\right) \times R S\left(B_{2}\right)\right)$ so

$$
h(k(0)) \bigcap \widetilde{T}\left(h\left(k\left(0_{1}\right)\right) \times h\left(k\left(0_{2}\right)\right)\right)
$$

is compact in this topology. Moreover,

$$
T^{-1}\left(h(k(0)) \cap \widetilde{T}\left(h\left(k\left(0_{1}\right)\right) \times h\left(k\left(0_{2}\right)\right)\right)\right)
$$

is a Hausdorff space with interior :

$$
\begin{aligned}
\left(M_{1}, M_{2}\right) \in 0_{1} \times 0_{2} & \Longrightarrow \widetilde{T}\left(M_{1}, M_{2}\right) \in h(k(0)) \cap \widetilde{T}\left(h\left(k\left(0_{1}\right)\right) \times h\left(k\left(0_{2}\right)\right)\right. \\
& \Longrightarrow\left(M_{1}, M_{2}\right) \in \widetilde{T}^{-1}\left(h(k(0)) \cap \widetilde{T}\left(h\left(k\left(0_{1}\right)\right) \times h\left(k\left(0_{2}\right)\right)\right)\right) .
\end{aligned}
$$

It follows that $\left.\widetilde{T}\right|_{\left.\widetilde{T}-1 \cdot h(k(0)) \cap \widetilde{T}\left(h\left(k\left(0_{1}\right)\right) \times h\left(k\left(0_{0}\right)\right)\right)\right)}$ is continuous so that $\left.\widetilde{T}\right|_{0_{1} \times 0_{2}}$ is continuous. $0_{1} \times 0_{2}$ is open; we have shown that $\widetilde{T}$ is a homeomorphism.

If $\widetilde{T}$ is surjective, then clearly $R S\left(B_{1} \otimes_{r} B_{2}\right)$ is Hausdorff. If $M=\widetilde{T}\left(M_{1}, M_{2}\right) \in R S\left(B_{1} \otimes_{r} B_{2}\right)$ then there are open sets $O_{i}$ with regular kernels such that $M_{i} \in O_{i}(i=1,2) . \quad \widetilde{T}\left(0_{1} \times 0_{2}\right)=0$ is an open set containing $M$ and $k(0)$ is regular by the first part of the above argument.

Consequently, $B_{1} \otimes_{\gamma} B_{2}$ is completly regular.

Theorem 2.8 will be used in Theorem 2.10. Just as in the numerical case it is of importance in our proof of the Wienertauberian theorem to be able to conclude that the elements vanishing outside compact sets in the strong structure space are dense. This notion will be made precise by means of the following definiton [21].

Definition 2.9. Let $B$ be a Banach algebra; for $\mathrm{S} \subset R S(B)$ define

$$
\begin{aligned}
J(S, B)= & \{x \in B ; x(M)=0 \in B / M \\
& \text { for all } M \text { in some open set containing } S\}
\end{aligned}
$$

and

$$
\begin{aligned}
J(\infty, B)= & \{x \in B ; x(M)=0 \in B / M \\
& \quad \text { for every } M \text { outside some compact set in } R S(B)\} .
\end{aligned}
$$

If no confusion seems likely, we use the notation $J(S)$ and $J(\infty)$. 
Theorem 2.10. Let $B_{1}$ and $B_{2}$ be completely regular Banach algebras and suppose $\widetilde{T}$ is bijective. Using - to denote norm closure we have the following implication:

$$
\begin{array}{rlr}
J\left(\infty, B_{i}\right)^{-} & =B_{i} & (i=1,2) \\
J\left(\infty, B_{i} \otimes{ }_{\gamma} B_{2}\right)^{-} & =B_{i} \otimes{ }_{\gamma} B_{1} . &
\end{array}
$$

Proof. The proof of this statement is not hard. Since $B_{1} \otimes B_{2}$ is dense in $B_{1} \otimes_{\gamma} B_{2}$ it suffices to consider elements of the form $x \otimes y$. So let $\varepsilon>0$ and $x \otimes y \in B_{1} \otimes B_{2}$ be given. From the assumptions we get that $\exists x_{1} \in B$ and a compact set $F_{1} \subset R S\left(B_{1}\right)$ such that $x_{1}\left(M_{1}\right)=0$ for $M_{1} \notin F_{1}$ and such that $\left|x-x_{1}\right|<\varepsilon$. Similarly, $\exists y_{1} \in B_{2}$ and a compact set $F_{2} \subset R S\left(B_{2}\right)$ such that $y_{1}\left(M_{2}\right)=0$ for $M_{2} \notin F_{2}$ and $\left|y-y_{1}\right|<\varepsilon$. $\gamma$ being a crossnorm we get immediately that

$$
\gamma\left(x \otimes y-x_{1} \otimes y_{1}\right) \leqq \varepsilon(|x|+|y|+\varepsilon) .
$$

Now, since $B_{1}$ and $B_{2}$ are completely regular

$$
\widetilde{T}: R S(B) \times R S\left(B_{2}\right) \longrightarrow R S\left(B_{1} \otimes_{\gamma} B_{2}\right)
$$

is continuous (Theorem 2.8) so $\widetilde{T}\left(F_{1} \times F_{2}\right)$ is compact.

If $M \notin \widetilde{T}\left(F_{1} \times F_{2}\right)$ and $M=\widetilde{T}\left(M_{1} M_{2}\right)$ then it follows first that $M_{1} \notin F_{1} \quad$ or $\quad M_{2} \notin F_{2}$ and next that $x_{1} \otimes y_{1}(M)=x_{1}\left(M_{1}\right) \otimes y_{1}\left(M_{2}\right)=0$. From this the theorem follows.

Now we are ready to combine the three concepts mentioned so far (tauberianism, strong semi-simplicity and complete regularity) in a proof of a ideal theoretic formulation of Wiener's tanberian theorem.

THEOREM 2.11. Let $G$ be a locally compact group such that $L^{1}(G)$ is strongly semi-simple, tauberian and completely regular and let $A$ be a strongly semi-simple, completely regular, tauberian Banach algebra. Suppose $\widetilde{T}$ is bijective. Then $L^{1}(G, A)$ is strongly semisimple, completely regular and tauberian.

Proof. Theorems 2.8 and 2.6. show that $L^{1}(G, A)$ is strongly semi-simple and completely regular. In [21] it is shown that under these circumstances $J\left(\infty, L^{1}(G, A)\right)^{-}$is the uniquely determined minimal closed 2-sided ideal with empty hull [21, Th. 1.2]. By the same token, $J(\infty, A)^{-}$is a closed 2 -sided ideal with empty hull. Since $A$ is tauberian $J(\infty, A)^{-}$cannot be proper, i.e., $J(\infty, A)^{-}=A$. Similarly,

$$
J\left(\infty, L^{1}(G)\right)^{-}=L^{1}(G) .
$$

But then (Theorem 2.10) $J\left(\infty, L^{1}(G, A)\right)^{-}=L^{1}(G, A)$, i.e., no closed proper 2-seided ideal in $L^{1}(G, A)$ has an empty hull. Thus $L^{1}(G, A)$ is tauberian. 
$\widetilde{T}$ is bijective if $G$ is locally compact and abelian [12]. Consequently, we also have

CoRollary 2.12. [20]. If $G$ is a locally compact abelian group and $C$ is a compact group, then the conclusion of Theorem 2.11 holds for $L^{1}(G \times C)$.

Proof. $L^{1}(C)$ is strongly semi-simple, completely regular and tauberian [21].

3. Spectral synthesis. A well known interpretation of 2.11 views it as an answer to a special case of the question of spectral synthesis : is a closed 2-sided ideal in a Banach algebra the intersection of the maximal regular 2-sided ideals containing it? Using the hull-kernel terminology and notation, Theorem 2.11 says that with certain conditions on $L^{1}(G)$ and $A$ our ideal $I \subset L^{1}(G, A)$ satisfies $I=k(h(I))$ if $h(I)=\varnothing$ (because then $I=L^{1}(G, A)$ ).

If $I=k(h(I))$ for every closed 2-sided ideal in a Banach algebra $B$ we say that $B$ admits spectral synthesis. Schwartz (in [17]) has shown that $L^{1}\left(E^{3}\right)$ does not admit spectral synthesis. In this section we shall prove some results along these lines for $L^{1}(G, A)$. The main result is that if $A$ does not admit spectral synthesis then $L^{1}(G, A)$ does not, either.

Lemma 3.1. Let $G$ be a locally compact group and $A$ a Banach algebra. Let $I$ be a closed 2-sided ideal in $A$ and define

$$
J_{I}=\left\{f \in L^{1}(G, A) ; f(g) \in I \text { a.e. } g \in G\right\}
$$

$J_{I}$ is a closed 2-sided ideal.

REMARK 3.2. The definition of $J_{I}$ should be interpreted as follows: Pick a function representative $f^{\prime}$ of the equivalence class of functions $f \in L^{1}(G, A)$; if $f^{\prime}(g) \in I$ for a.e. $g \in G$ then $f \in J_{I}$.

Proof of Lemma 3.1. Clearly $J_{I}$ is a subspace. If $f_{n} \rightarrow f$ in $L^{1}(G, A)$-norm then there is a subsequence $f_{n_{k}}$ converging to $f$ a.e. Since $I$ is closed this implies that $J_{I}$ is closed.

Consequently, to show that $J_{I}$ is an ideal we need only check the $A$-translation invariance of $J_{I}$ (Theorem 2.2). (Note that no approximate identity is needed when we use Theorem 2.2 here.) But the $A$-translation invariance of $J_{I}$ is an immediate consequence of the definition of $J_{I}$ and the fact that $I$ is an ideal. 
REMARK 3.3. Clearly, in Lemma 3.1, "2-sided" could be replaced by "left" or "right".

LEMMA 3.4. With the assumptions of Lemma 3.1, let $I$ be a proper closed 2-sided ideal in $A$ and $J_{I}$ the corresponding ideal in $L^{1}(G, A)$.

Then

$$
\widetilde{T}\left(R S\left(L^{1}(G)\right) \times h(I)\right) \subseteq h\left(J_{I}\right) .
$$

If $\widetilde{T}$ is bijective, then

$$
h\left(J_{I}\right)=\widetilde{T}\left(R S\left(L^{1}(G)\right) \times h(I)\right)
$$

so that

$$
k\left(h\left(J_{I}\right)\right)=J_{I} \Longrightarrow k(h(I))=I \text {. }
$$

Proof. We shall show that if $I_{1} \subseteq L^{1}(G)$ is a modular 2-sided ideal and if $I_{2} \supseteqq I$ is a modular 2-sided ideal in $A$, then $T\left(I_{1} I_{2}\right) \supseteqq J_{I}$ :

We make the following observations: If $I^{\prime \prime}$ is any closed 2-sided ideal in $A$, then we can consider $L^{1}\left(G, A / I^{\prime \prime}\right)$. Any $f \in L^{1}(G, A)$ induces a mapping $f \rightarrow f_{I^{\prime \prime}}$ by

$$
f_{I^{\prime \prime}}(g)=f(g) / I^{\prime \prime} \quad \text { a.e. } g \in G \text {. }
$$

Clearly,

$$
J_{I^{\prime \prime}}=\left\{f \in L^{1}(G, A) \mid f_{I^{\prime \prime}}=0\right\} .
$$

If $I^{\prime}$ is a closed 2-sided ideal in $L^{1}(G)$ and $B$ is any Banach algebra, we have a mapping

$$
\alpha_{I^{\prime}}^{B}: L^{1}(G) \otimes_{\gamma} B \longrightarrow L^{1}(G) / I^{\prime} \otimes_{\gamma} B
$$

i.e., a mapping

$$
L^{1}(G, B) \longrightarrow L^{1}(G) / I^{\prime} \otimes_{r} B
$$

defined by

$$
\alpha_{I^{\prime}}^{B}\left(\sum x_{i} \otimes y_{i}\right)=\sum x_{i} / I^{\prime} \otimes y_{i}
$$

for any $\sum x_{i} \otimes y_{i} \in L^{1}(G, B)$.

If $\left(I^{\prime}, I^{\prime \prime}\right)$ is a pair of modular ideals, then

$$
T\left(I^{\prime}, I^{\prime \prime}\right)=\operatorname{kernel}\left(\alpha_{I^{\prime}}^{A / I^{\prime \prime}} \circ\left(f \longrightarrow f_{I^{\prime \prime}}\right)\right) \text {. }
$$

Now the above claim follows easily: If $I_{1} \subset L^{1}(G)$ is a modular 2-sided ideal and $I_{2} \supset I$ is a modular ideal, then 


$$
\begin{aligned}
f \in J_{I} & \Longrightarrow f \in \operatorname{kernel}\left(f \longrightarrow f_{I}\right) \\
& \Longrightarrow f \in \operatorname{kernel}\left(f \longrightarrow f_{I_{2}}\right) \\
& \Longrightarrow f \in \operatorname{kernel}\left(\alpha_{I_{1}}^{A / I_{1}} \circ\left(f \longrightarrow f_{I_{2}}\right)\right) \\
& \Longrightarrow f \in T\left(I_{1}, I_{2}\right) .
\end{aligned}
$$

With this proved, the lemma follows from the general properties of $T$ and $\widetilde{T}$ :

If $M_{2} \in h(I), M_{1} \in R S\left(L^{1}(G)\right)$ then

$$
\widetilde{T}\left(M_{1}, M_{2}\right) \supseteqq T\left(M_{1}, M_{2}\right) \supseteqq J_{I}
$$

so

$(*)$

$$
\widetilde{T}\left[R S\left(L^{1}(G)\right) \times h(I)\right] \subseteq h\left(J_{I}\right) .
$$

If $\widetilde{T}$ is bijective, then it is a simple matter to show that if $M_{3} \in h\left(J_{I}\right)$, then

$$
\widetilde{T}^{-1}\left(M_{3}\right)=\left(M_{1}, M_{2}\right)
$$

has the property that $M_{2} \supseteqq I$, so that

$$
\widetilde{T}^{-1}\left(h\left(J_{I}\right)\right) \subseteq R S\left(L^{1}(G)\right) \times h(I) .
$$

Combining $(*)$ and $(* *)$ the second claim of the lemma follows:

$$
h\left(J_{I}\right)=\widetilde{T}\left(R S\left(L^{1}(G)\right) \times h(I)\right) .
$$

To get the last statement of the lemma, we replace $I$ by $l(h(I))$ in $(* * *)$ :

$$
\begin{aligned}
h\left(J_{k(h(I))}\right) & =T\left(R S\left(L^{1}(G)\right) \times h(k(I))\right) \\
& =T\left(R S\left(L^{1}(G)\right) \times h(I)\right) \\
& =h\left(J_{l}\right) .
\end{aligned}
$$

Therefore

$$
\begin{aligned}
k\left(h\left(J_{k(h(I))}\right)\right) & =k\left(h\left(J_{I}\right)\right) \\
& \supseteqq J_{k(h(I))} \supseteqq J_{J^{\prime}},
\end{aligned}
$$

from which we conclude immediately that if

$$
k\left(h\left(J_{l}\right)\right)=J_{I}
$$

then

$$
k(h(I))=I .
$$

The following corollaries are straightforward. 
CoRollary 3.5. If $\widetilde{T}$ is bijective and if $I$ is a closed 2-sided ideal in $A$ which is contained in no maximal regular ideal then the same is true about $J_{I}$.

Corollary 3.6. If $\widetilde{T}$ is bijective, then $L^{1}(G, A)$ does not admit spectral synthesis if $A$ does not.

CoRollary 3.7. Let $G$ be a locally compact group and $E^{n} n$ dimensional Euclidean space (considered an additive group). $L^{1}\left(G \times E^{n}\right)$ does not admit spectral synthesis if $n \geqq 3$.

Proof. [17], Proposition 1.4, and Corollary 3.6.

If we add the assumption that $G$ be abelian, Lemma 3.5 can be strengthened.

Lemma 3.8. Suppose $G$ is a locally compact abelian group and $A$ is a Banach algebra. If $I$ is a closed proper 2-sided ideal, then

$$
k\left(h\left(J_{I}\right)\right)=J_{I} \text { if and only if } k(h(I))=I .
$$

Proof. It is not difficult to show that if $M_{1}$ is a maximal regular ideal in $L^{1}(G)$ and $\chi$ the corresponding character on $G$ then $M_{1}$ defines. a mapping

$$
\varphi_{M_{1}}: L^{1}(G, A) \longrightarrow A
$$

given by

$$
\varphi_{M_{1}}\left(\sum x_{i} \otimes y_{i}\right)=\sum_{1}\left(x_{i}\right) y_{i}
$$

for every $\sum x_{i} \otimes y_{i} \in L^{1}(G) \otimes{ }_{r} A=L^{1}(G, A)$ and that this mapping has the integral expression

$$
\varphi_{M_{1}}(f)=\int_{G} f(g) \chi(g) d g \quad \text { for all } f \in L^{1}(G, A) .
$$

Next, we note that if $M \in R S\left(L^{1}(G, A)\right)$ and $\widetilde{T}(M)=\left(M_{1}, M_{2}\right)$, then

$$
f \in M \Longleftrightarrow \varphi_{M_{1}} f \in M_{2} \text {. }
$$

To complete the proof we must show that

$$
f \in k\left(\left\{\widetilde{T}\left(M_{1}, M_{2}\right) ; M_{2} \in h(I)\right\}\right) \Longrightarrow f \in J_{I}
$$

under the assumption that $k(h(I))=I$. Using the definition of $J_{I}$ and $k(h(I))=I$ we see that we must prove the following implication:

$$
\begin{gathered}
\varphi_{M_{1}}(f) \in I \text { for any } M_{1} \in R S\left(L^{1}(G)\right) \\
\Longrightarrow f(g) \in I \text { for a.a. } g \in G .
\end{gathered}
$$


So, suppose

$$
\varphi_{M_{1}}(f)=\int_{G} f(g) \chi(g) d g \in I
$$

for every character $\chi$ on $G$. By means of the approximation theorem for abelian groups (cf.e.g., [14, Corollary 4, p. 406]) we get that

$$
\int f(g) \psi(g) d g \in I
$$

for every continuous function $\psi: G \rightarrow \mathbf{C}$ with compact support. If $f$ is continuous a well known argument will show that $f(g) \in I$ for every $g \in G$.

To cover the general case we let $\left\{u_{\alpha}\right\}$ be an approximate identity in $L^{1}(G)$ consisting of continuous functions with compact support. Using standard results from the numerical case and Proposition 1.2. we conclude first that $u_{\alpha} * f \rightarrow f$ in $L^{1}(G, A)$-norm and next that $u_{\alpha} * f$ is continuous for every $\alpha$. From $\left\{u_{\alpha} * f\right)$ we can pick a sequence and from this a subsequence converging a.e. to $f$. Consequently, it suffices to show that $\left(u_{\alpha} * f\right)(g) \in I$ for every $g \in G$. But

$$
\begin{aligned}
u_{\alpha} * f(g) & =\int_{\theta} u_{\alpha}\left(g_{1}\right) f\left(g_{1}^{-1} g\right) d g_{1} \\
& =\int_{G} u_{\alpha}\left(g h^{-1}\right) f(h) d h \in I
\end{aligned}
$$

because of $(*)$. This completes the proof.

Just as in [8] we can extract the following corollaries.

Corollary 3.9. If $G$ is abelian and $A$ is a Banach algebra, the only maximal regular 2-sided ideals containing $J_{M_{2}}$ with $M_{2} \in R S(A)$ are of the form $\widetilde{T}\left(M_{1} M_{2}\right)$ where $M_{1}$ is an arbitrary regular maximal 2-sided ideal in $L^{1}(G)$.

Corollary 3.10. Same assumptions as in Corollary 3.9. Suppose $M_{2}^{\prime}, M_{2}^{\prime \prime} \in R S(A)$ and $M_{2}^{\prime} \neq M_{2}^{\prime \prime}$; then

$$
\left(\widetilde{T}\left(M_{1}, M_{2}^{\prime}\right)+J_{M_{2}^{\prime \prime}}\right)^{-}=L^{1}(G, A)
$$

(where - denotes norm closure) for any $M_{1} \in R S\left(L^{1}(G)\right.$ ).

4. On sets of spectral synthesis. Turning to more positive assertions about spectral synthesis we shall study $L^{1}(G, A)$ in the case where $L^{1}(G)$ and $A$ are strongly semi-simple and completely regular. The situation in which a certain weak form of spectral synthesis holds will dominate this section. 
Definition 4.1. Let $A$ be an algebra. A closed set $S \subset R S(A)$ is said to be a set of spectral synthesis if $S$ uniquely determines a closed ideal $I$ such that $h(I)=S$. Clearly then $I=k(S)$, i.e., $I=k(h(I))$.

Definition 4.2. A Banach algebra $A$ in which singleton sets in $R S(A)$ are sets of spectral synthesis is called an $N^{*}$-algebra [21]. An ideal $I$ for which $h(I)$ consists of just one point is a primary ideal. Consequently, an $N^{*}$-algebra is a Banach algebra in which closed primary ideals are maximal regular.

The following proposition generalizes Theorem 4 in [8] and will be used to investigate conditions under which $L^{1}(G, A)$ is an $N^{*}$-algebra.

Proposition 4.3. Let $G$ be a locally compact group and $A$ a commutative Banach algebra. Suppose $I \subset L^{1}(G, A)$ is a closed 2-sided ideal such that $I \supset I_{M_{0}}$ for some $M_{0} \in R S(A)$. Let $h(I)=\left\{\widetilde{T}\left(M^{\prime}, M^{\prime \prime}\right)\right\}$. If $W=\left\{M^{\prime} ; \widetilde{T}\left(M^{\prime}, M^{\prime \prime}\right) \in h(I)\right.$ for some $\left.M^{\prime \prime} \in R S(A)\right\}$ is a set of spectral synthesis then $h(I)$ is a set of spectral synthesis, i.e. $I=k(h(I))$.

Proof. From Lemma 3.4 (i) we get that

$$
\begin{aligned}
h(I) \subset h\left(I_{M_{0}}\right) & \subset\left\{\widetilde{T}\left(M_{1}, M_{2}\right) ; M_{2} \in h\left(M_{0}\right), M_{1} \in R S\left(L^{1}(G)\right)\right\} \\
& =\left\{\widetilde{T}\left(M_{1}, M_{0}\right) ; M_{1} \in R S\left(L^{1}(G)\right)\right\} . \\
\text { i.e., } h(I) & =\widetilde{T}\left(W, M_{0}\right)
\end{aligned}
$$

with a slight abuse of notation.

Now, using the notation of the proof of Lemma $3.8 M_{0} \in R S(A)$ defines a homomorphism

$$
\varphi_{M_{0}}: L^{1}(G, A) \rightarrow L^{1}(G)
$$

by

$$
f=\sum x_{i} \otimes y_{i} \in L^{1}(G, A) \Rightarrow \varphi_{M_{0}} f=\sum M_{0}\left(y_{i}\right) x_{i} \in L^{1}(G) .
$$

We consider the image of $I$ under $\varphi_{M_{0}}$, i.e., let

$$
K=\varphi_{M_{0}}(I) \text {. }
$$

We wish to show that $K$ is a closed 2 -sided ideal in $L^{1}(G)$. Since $\varphi_{M_{0}}$ is a homomorphism onto it is clear that $K$ is an ideal; to show that $K$ is closed we prove that if $u_{0}$ is an identity modulo $M_{0}$ then

$$
K=\left\{x \in L^{1}(G) ; x \otimes u_{0} \in I\right\} .
$$

If this equation holds it is elementary to show that $K$ is closed: $\left\{x_{n}\right\} \subset K, x_{n} \rightarrow x_{0} \Rightarrow x_{n} \otimes u_{0} \rightarrow x_{0} \otimes u_{0} \in I$ (since $I$ is closed) $\Rightarrow x_{0} \in K$. 
So suppose $x \otimes u_{0} \in I$; then $\varphi_{M_{0}}\left(x \otimes u_{0}\right)=x \in K$. Conversely, $x \in K \Rightarrow \exists f \in I$ such that $\varphi_{M_{0}} f=x$. Consider $f-x \otimes u ; f(g)-x(g) u_{0} \in M_{0}$ for a.a.g $\in G$ since if $f=\sum x_{i} \otimes y_{i}$ then

$$
\begin{aligned}
& M_{0}\left(f(g)-x(g) u_{0}\right) \\
& \quad=\sum x_{i}(g) M_{0}\left(y_{i}\right)-M_{0}\left(y_{i}\right) x_{i}(g)=0
\end{aligned}
$$

From the definition of $I_{M_{0}}$ it follows that $f-x \otimes u_{0} \in I_{M_{0}}$, i.e., $f-x \otimes u_{0} \in I$ (note, incidentally, that $f-\varphi_{M_{0}} f \otimes u_{0} \in I_{M_{0}}$ for every $\left.f \in L^{1}(G, A)\right)$. But since $f \in I$ it follows that $x \otimes u_{0} \in I$. This proves that $K=\left\{x \in L^{1}(G) ; x \otimes u_{0} \in I\right\}$, and thus that $K$ is a closed 2-sided ideal (not necessarily $\neq\{0\}$ ).

The above characterization of $K$ together with the definition of the mapping $\widetilde{T}$ readily yield the fact that $h(K)=W$. But since $f \in I$ if and only if $\varphi_{M_{0}} f \in K=k(W)$ it follows that $f \in I$ if and only if $M \otimes M_{0}(f)=M\left(_{M_{0}} f\right)=0$ for every $M \in W$, i.e., $f \in I$ if and only if $f \in k(h(I))$. This proves the proposition.

Using proposition 4.3. we can obtain a sufficient condition for $L^{1}(G, A)$ to be an $N^{*}$-algebra.

THEOREM 4.4. Let $G$ be a locally compact group such that $L^{1}(G)$ is completely regular, strongly semi-simple and tauberian and let $A$ be a commutative, completely regular, (strongly) semi-simple Banach algebra. If $L^{1}(G)$ and $A$ are $N^{*}$-algebras, then so is $L^{1}(G, A)$.

Proof. We must show that if $I$ is a primary closed 2-sided ideal, e.g., $h(I)=\left\{M_{0}=\widetilde{T}\left(M_{0}^{\prime}, M_{0}^{\prime \prime}\right)\right\}$ then $I=M_{0}$. Since $L^{1}(G, A)$ is strongly semi-simple and completely regular (Theorems 2.6 and 2.10) we can assume without loss of generality that $I=\left(J\left(M_{0}\right) \cap J(\infty)\right)^{-}[21$, p. 180]. We wish to apply Proposition 4.3 ; consequently, we must show that $I \supset I_{M_{0}^{\prime \prime}}$.

So let $f \in I_{M_{0}^{\prime \prime}}$; we first show that we can approximate $f$ by means of continuous functions in $I_{M_{0}^{\prime \prime}} . \quad f \in I_{M_{0}^{\prime \prime}} \Rightarrow f(g) \in M_{0}^{\prime \prime}$ for a.a.g $\in G$. If $\left\{u_{\alpha}\right\}$ is an approximate identity in $L^{1}(G)$ consisting of continuous functions then

$$
u_{\alpha}^{*} f(\cdot)=\int_{G} u_{\alpha}\left(g^{\prime}\right) f\left(g^{\prime-1} \cdot\right) d g^{\prime}
$$

is continuous and

$$
u_{\alpha}^{*} f \rightarrow f
$$

as $\alpha \rightarrow \infty$.

Moreover, since $\mathrm{f}(g) \in M_{0}^{\prime \prime}$ for $\quad$ a.a.g. $\in G, \quad u_{\alpha}\left(g^{\prime}\right) f\left(g^{\prime-1} g\right) \in M_{0}^{\prime \prime}$ for 
a.a.g, $g^{\prime} \in G$. An approximation argument similar to the one employed in the proof of Theorem 2.2. then yields that $u_{\alpha}^{*} f(g) \in M_{0}^{\prime \prime}$ for a.a.g $\in G$, i.e., $u_{r}^{*} f \in I_{M \alpha_{0}^{\prime \prime}}$.

Since we want to show that $f \in I_{M_{0}^{\prime \prime}} \Rightarrow f \in I$ and since $I$ is closed we can assume therefore, without any loss of generality, $f \in I_{M_{0}^{\prime \prime}}$ to be continuous.

So let $f \in I_{M_{0}^{\prime \prime}}$ be a given continuous function and let also $\varepsilon>0$ be given. $\exists$ compact set $E \subset G$ such that

$$
\int_{G \backslash E}|f(g)| d g<\frac{\varepsilon}{2} .
$$

Let $g_{0} \in E$ and consider $f\left(g_{0}\right) \in M_{0}^{\prime \prime}\left(f(g) \in M_{0}^{\prime \prime}\right.$ for every $g \in G$ since $f$ is continuous). $A$ is assumed to be a semi-simple completely regular $N^{*}$-algebra so $\exists y_{g_{0}}$ such that

$$
\left|f\left(g_{0}\right)-y_{g_{0}}\right|<\frac{\varepsilon}{2 \mu(E)}
$$

and $M^{\prime \prime}\left(y_{g_{0}}\right)=0$ for all $M^{\prime \prime}$ in a neighborhood $N_{g_{0}}$ of $M_{0}^{\prime \prime}$ and outside a compact set $C_{g_{0}} \subset R S(A)$. Let

$$
S_{g_{0}}=\left\{g ;\left|f(g)-y_{g_{0}}\right|<\frac{\varepsilon}{2 \mu(E)}\right\} .
$$

Since $f$ is continuous $S_{g_{0}}$ is an open neighborhood of $g_{0}$. Clearly, $\left\{S_{g_{0}}, g_{0} \in E\right\}$ is an open cover of $E$ and so has a finite subfamily covering $E$.

Let the open sets covering $E$ be $\left\{S_{i}\right\}_{i=1}^{n}$, let the appropriate points $y_{g_{0}}$ be $\left\{y_{i}\right\}_{r=1}^{n}$ and let the corresponding neighborhoods and compact sets in $R S(A)$ be $\left\{N_{i}\right\}_{i=1}^{n}$ and $\left\{C_{i}\right\}_{i=1}^{n}$, respectively. Define

$$
\begin{aligned}
& T_{1}=S_{1} \cap E \\
& \left.T_{i}=\left(S_{i}\right)\left(\bigcup_{j<i} S_{j}\right)\right) \cap E \quad i=2, \cdots, n ;
\end{aligned}
$$

then $T_{i} \cap T_{j} \varnothing$ if $i \neq j$ and $\bigcup_{i=1}^{n} T_{i}=E$. Let $1_{T_{i}}$ be the characteristic function of $T_{i}(i=1, \cdots, n)$. If we define

$$
f^{\prime}=\sum_{i=1}^{n} 1_{T_{i}} \otimes y_{i}
$$

then, clearly, $f^{\prime} \in L^{1}(G, A)$. Moreover, $M\left(f^{\prime}\right)=0$ for every

$$
M \in\left\{\widetilde{T}\left(M^{\prime}, M^{\prime \prime}\right) ; M^{\prime} \in R S\left(L^{1}(G)\right), M^{\prime \prime} \in\left(\bigcap_{\imath=1}^{n} N_{i}\right) \cup\left(R S(A) \backslash \bigcup_{i=1}^{n} C_{i}\right\}\right.
$$

i.e., $M\left(f^{\prime}\right)$ vanishes in a neighborhood of $M_{0}$. 


$$
\begin{gathered}
\left|f-f^{\prime}\right|=\int_{G}\left|f(g)-f^{\prime}(g)\right| d g=\int_{E}\left|f(g)-\sum_{i=1}^{n} 1_{T_{i}}(g) y_{i}\right| d g \\
\quad+\int_{G / E}|f(g)| d g<\sum_{i} \int_{T_{i}}\left|f(g)-\sum 1_{T_{i}}(g) y_{i}\right| d g+\varepsilon / 2 \\
=\sum_{i} \int_{T_{i}}\left|f(g)-y_{i}\right| d g+\varepsilon / 2, \quad \text { since } T_{i} \cap T_{j}=\varnothing \quad \text { if } \quad i \neq j \\
\sum_{i} \frac{\varepsilon}{2 \mu(E)} \mu\left(T_{i}\right)+\varepsilon / 2=\varepsilon .
\end{gathered}
$$

It remains to note that each $1_{I_{i}}$ can be approximated by an element $x_{i}$ from $J\left(\infty, L^{1}(G)\right)$, (because $L^{1}(G)$ is tauberian) i.e., $1_{T_{i}} \otimes y_{i}$ can be approximated by $x_{i} \otimes y_{i}(i=1, \cdots, n)$. Since the sum is finite it follows that $\sum x_{i} \otimes y_{i} \in J\left(\infty, L^{1}(G, A)\right) \cap J\left(M_{0}, L^{1}(G, A)\right)$.

We have shown that $I_{M_{0}} \subset I$. From Proposition 4.3. we get that since $\left\{M_{0}^{\prime}\right\}$ is a set of spectral synthesis $\left(L^{1}(G)\right.$ is an $N^{*}$-algebra) $I=k(h(I))=M_{0}$.

This proves that $L^{1}(G, A)$ is an $N^{*}$-algebra.

REMARK 4.5. In [10] it is shown that if $G$ is a locally compact abelian group and $C$ is a compact group then $L^{1}(G \times C)$ is an $N^{*}$ algebra. This result is contained in Theorem 4.4.

Incidentally, if the tensor product $B \otimes_{\gamma} C$ of two Banach algebras is an $N^{*}$-algebra then it is possible to give a direct description of the mapping $\widetilde{T}: R S(B) \times R S(C) \rightarrow R S\left(B \otimes{ }_{\gamma} C\right)$.

Propositron 4.6. Suppose $B \otimes{ }_{\gamma} C$ is an $N^{*}$-algebra, and suppose $\widetilde{T}$ is bijective. Let $M_{1} \in R S(B), M_{2} \in R S(C)$ and consider

$$
\begin{aligned}
& I_{M_{1}}=\left\{x \otimes y ; x \in M_{1}, y \in C\right\} \\
& I_{M_{2}}=\left\{x \otimes y ; x \in B, y \in M_{2}\right\} .
\end{aligned}
$$

Let $M=\widetilde{T}\left(M_{1}, M_{2}\right)$. If we use the symbol [ ] to denote the ideal generated by the set inside the brackets then

$$
M=\left[I_{M_{1}}+I_{M_{2}}\right]^{-} \text {. }
$$

Sketch of Proof. Because of the assumption on $B \otimes_{r} C$ it suffices to show that $\left[I_{M_{1}}+I_{M_{2}}\right]^{-}$is primary (clearly $\left[I_{M_{1}}+I_{M_{2}}\right]^{-} \subset M$ ). To show that $\left[I_{M_{1}}+I_{M_{2}}\right]^{-}$is a primary ideal a slight variation of the argument used in the proof of Lemma 4.7. can be used. Therefore, we omit all further details.

With the notation of Proposition 4.6. we have the following

Lemma 4.7. Suppose $B$ and $C$ are strongly semi-simple and 
completely regular Banach algebras, and suppose $\widetilde{T}$ is bijective. Let $M=\widetilde{T}\left(M_{1}, M_{2}\right) \in R S\left(B \otimes{ }_{i} C\right)$ be given. Then

$$
\begin{aligned}
I_{0} & =\left[J\left(M_{1}\right) \cap J(\infty, B) \otimes J(\infty, C)+J(\infty, B) \otimes J\left(M_{2}\right) \cap J(\infty, C)\right] \\
& =\left(J(M) \cap J\left(\infty, B \otimes{ }_{\gamma} C\right)\right) .
\end{aligned}
$$

Proof. Since $B$ and $C$ are completely regular $\widetilde{T}$ is a homeomorphism (Theorem 2.10). Therefore, if

$$
x \otimes y \in\left(J\left(M_{1}\right) \cap J(\infty, B)\right) \otimes J(\infty, C)
$$

it is clear that $x \otimes \mathrm{y} \in J\left(\infty, B \otimes_{\gamma} C\right)$. Moreover, $x \in J\left(M_{1}\right) \cap J(\infty, B) \subset$ $J\left(M_{1}\right) \Rightarrow \exists$ open neighborhood $N\left(M_{1}\right)$ such that $x\left(M_{1}^{\prime}\right)=0$ for all $M_{1}^{\prime} \in N\left(M_{1}\right)$. $\widetilde{T}\left(N\left(M_{1}\right) \times R S(C)\right)$ is an open neighborhood of $M$ in $R S\left(B \otimes{ }_{\gamma} C\right)$ and $x \otimes y\left(M^{\prime}\right)=x\left(M_{1}^{\prime}\right) \otimes y\left(M_{2}^{\prime}\right)=0$ for every

$$
M^{\prime}=\widetilde{T}\left(M_{1}^{\prime}, M_{2}^{\prime}\right) \in \widetilde{T}\left(N\left(M_{1}\right) \times R S(C)\right) .
$$

This shows that $J\left(M_{1}\right) \cap J(\infty, B) \otimes J(\infty, C) \subset J(M) \cap J\left(\infty, B \otimes{ }_{i} C\right)$. Repeating this argument we get that

$$
I_{0} \subset J(M) \cap J\left(\infty, B \otimes_{r} C\right) .
$$

Because of the remark at the bottom of p. 180 of [21] to prove equality it suffices to show that $I_{0}$ is a primary ideal. So suppose $x \otimes y \in M^{\prime}=\widetilde{T}\left(M_{1}^{\prime}, M_{2}^{\prime}\right)$ for all $x \in J\left(M_{1}\right) \cap J(\infty, B), y \in J(\infty, C)$. We can choose $y_{0} \in J(\infty, C)$ such that $y_{0} \notin M_{2}$ [21, Corollary 1.2.2.]. Since $x \otimes y_{0} \in M^{\prime}$ for all $x \in J\left(M_{1}\right) \cap J(\infty, B)$ it follows that $M_{1}^{\prime}=M_{1}$ (since $J\left(M_{1}\right) \cap J(\infty, B)$ is a primary ideal [21, Th. 1.2.]).

Repeating this argument for $J(\infty, B) \otimes J\left(M_{2}\right) \cap J(\infty, C)$ ) we get that $M_{2}=M_{2}^{\prime}$ and therefore that $I_{0}$ is primary.

[21] has transferred Ditkin's condition, condition (D) to the noncommutative case. Here we shall consider the following version.

Definition 4.8. A Banach algebra $B$ satisfies condition (D) if for every $M \in R S(\widetilde{B})$ ( $M$ may be all of $B) \exists$ bounded net $\left\{h_{\alpha}\right\} \subset J(M) \cap J(\infty)$ such that for every $f \in(J(M) \cap J(\infty))^{-} \exists$ subnet $\left\{h_{\alpha}^{\prime}\right\}$ such that $f h_{\alpha}^{\prime} \rightarrow f$.

Lemma 4.9. Let $B$ and $C$ be strongly semi-simple completely regular Banach algebras, and suppose $\widetilde{T}$ is bijective. If $B$ and $C$ satisfy condition $(D)$, then so does $B \otimes{ }_{i} C$.

Proof. First, let $M \in R S\left(B \otimes{ }_{\gamma} C\right), M=\widetilde{T}\left(M_{1}, M_{2}\right)$. By assumption $\exists$ bounded nets $\left\{h_{M_{i}}\right\} \subset J\left(M_{i}\right) \cap J(\infty)$ so that for every $x_{i} \in J\left(M_{i}\right) \cap J(\infty)$ $\exists$ subnet $\left\{h_{M_{i}}^{\prime}\right\}$ for which $x_{i} h_{M_{i}}^{\prime} \rightarrow x ;(i=1,2)$. Moreover, $\exists$ bounded 
nets $\left\{h^{\infty 1}\right\} \subset J(\infty, B),\left\{h^{\infty 2}\right\} \subset J(\infty, C)$ so that $x h_{x}^{\infty i} \rightarrow x$ for every $x \in J(\infty, B)$ or $J(\infty, C)$ (where $\left\{h_{x}^{\infty i}\right\}$ is a subnet of $\left\{h^{\infty i}\right\}$ ). The net we shall use in $B \otimes C$ is $\left\{k^{1} \otimes k^{2}\right\}$ where $k^{i}$ is defined as follows.

$$
\begin{aligned}
& k^{1} \in\left\{h_{M_{1}}\right\} \Rightarrow k^{2} \in\left\{h_{M_{2}}\right\} \quad \text { or } \quad\left\{h^{\infty 2}\right\} \\
& k^{1} \in\left\{h^{\infty 1}\right\} \Rightarrow k^{2} \in\left\{h^{\infty 2}\right\} .
\end{aligned}
$$

It is not difficult to see that $\left\{k^{1} \otimes k^{2}\right\} \subset J(M) \cap J\left(\infty, B \otimes_{\gamma} C\right)$. Because $\left\{k^{1} \otimes k^{2}\right\}$ is uniformly bounded and because of Lemma 4.7 it suffices to consider elements of the form $x \otimes y$ with, say, $x \in J\left(M_{1}\right) \cap J(\infty, B)$ and $y \in J(\infty, C)$. Corresponding to this element we pick the appropriate subnets $\left\{h_{M_{i}}^{\prime}\right\} \subset\left\{h_{M_{i}}\right\},\left\{h^{\infty}\right\} \subset\left\{h^{\infty 2}\right\}$ and see immediately that

$$
(x \otimes y)\left(h_{M_{1}}^{\prime} \otimes h^{\infty_{2}^{\prime}}\right)=x h_{M_{1}}^{\prime} \otimes y h^{\infty_{2}^{\prime}} \rightarrow x \otimes y .
$$

A slight variation of this argument will handle the case $M=B \otimes{ }_{r} C$.

We are now ready to state the strongest result concerning spectral synthesis that we know of in this context. Being essentially an application of Corollary 2.6.1 in [21] we shall simply state it as

Corollary 4.10. Let $G$ be a locally compact group and $A$ a Banach algebra so that $L^{1}(G)$ and $A$ are strongly semi-simple, completely regular and satisfy condition $(D)$. Assume further that $L^{1}(G, A)$ is an $N^{*}$-algebra, and that $\widetilde{T}$ is bijective. Let $I$ be a closed 2-sided ideal in $L^{1}(G, A)$. If the boundary of $h(I)$ in $R S\left(L^{1}(G, A)\right)$ contains no perfect set $(\neq \varnothing)$, then $I=k(h(I))$.

Proof. By Theorem 2.6, Theorem 2.8 and Lemma $4.9 L^{1}(G, A)$ fulfils the hypotheses in Corollary 2.6.1 of [21]. Therefore the conclusion follows.

5. Concluding remarks. A comparison between this paper and [9] or [8] will quickly reveal some similarities. Both of these papers, however, deal with commutative algebras exclusively. While [8] has served as a starting point for the investigations of §3, [9] was unknown to the author at the time $\$ 2$ was being developed.

\section{BIBLIOGRAPHY}

1. Dixmier, Les $C^{*}$-algebres et leur representations, Gauthier-Villars, Paris 1964.

2. Gelbaum, Note on the tensor product of Banach algebras, Proc. Amer. Math. Soc. 12 (1961), 750-757.

3. - Tensor products and related questions, Trans. Amer. Math. Soc. 103 (1962), 525-548.

4. - Tensor products of Banach algebras (submitted to the Proc. Amer. Math. Soc.) 
5. Grothendieck, Produit tensoriels topologiquis et espaces nucleaires, Mem. Amer. Math. Soc. 16 (1955).

6. Grove, A generalized group algebra for compact groups, Studia Math. 26(1965), 73-90.

7. Hausner, The tauberian theorem for group algebras of vector-valued functions, Pacific J. Math. 7 (1957), 1603-1610.

8. — On generalized group algebras, Proc. Amer. Math. Soc. 10 (1959), 1-10. 9. Johnson, Spaces of functions with values in a Banach algebra, Trans. Amer. Math. Soc. 92 (1959), 411-429.

10. Kaplansky, Primary ideals in group algebras, Proc. Nat. Acad. Sci. U.S.A. 35 (1949), 133-136.

11. K. B. Laursen, Tensor products Banach algebras with involution (to appear in Trans. Amer. Math. Soc. 136 (1969), 467-487.

12. Maximal two-sided ideals in tensor products of Banach algebras (submitted to the Proc. Amer. Math. Soc.)

13. Loomis, An introduction to abstract harmonic analysis, Van Nostrand, Princeton, 1953.

14. Naimark, Normed rings, rev. ed. P. Noordhoff, Ltd., Groningen, 1964.

15. Rickart, General theory of Banach algebras, Van Nostrand, Princeton, 1960.

16. Schatten, A theory of cross spaces, Princeton Univ. Press, Princeton, 1950.

17. Schwartz, Sur une propriete de synthese spectrale dans les groupes non-compacts,

C. R. Acad. Sci. Paris 227 (1948) 424-426.

18. Segal, Group algebra of a locally compact group, Trans. Amer. Math. Soc. 61 (1947), 69-105.

19. Spicer, Group algebras of vector-valued functions, thesis, Univ. of Minn., 1965.

20. Willcox, Note on certain group algebras, Proc. Amer. Math. Soc. 7, (1956) 874879.

21. Some structure theorems for a class of Banach algebras, Pacific J. Math. 6 (1956), 177-192.

Received June 8, 1968, and in revised form March 18, 1969

UNIVERSITY OF AARHUS

DENMARK 


\section{PACIFIC JOURNAL OF MATHEMATICS}

\section{EDITORS}

H. ROYDEN

Stanford University

Stanford, California

Richard Pierce

University of Washington

Seattle, Washington 98105
J. DugundJI

Department of Mathematics

University of Southern California

Los Angeles, California 90007

BASIL GORDON

University of California

Los Angeles, California 90024

\section{ASSOCIATE EDITORS}

E. F. BECKENBACH

B. H. NeUmanN

F. WOLF

K. YOSHIDA

\section{SUPPORTING INSTITUTIONS}

UNIVERSITY OF BRITISH COLUMBIA

CALIFORNIA INSTITUTE OF TECHNOLOGY

UNIVERSITY OF CALIFORNIA

MONTANA STATE UNIVERSITY

UNIVERSITY OF NEVADA

NEW MEXICO STATE UNIVERSITY

OREGON STATE UNIVERSITY

UNIVERSITY OF OREGON

OSAKA UNIVERSITY

UNIVERSITY OF SOUTHERN CALIFORNIA
STANFORD UNIVERSITY

UNIVERSITY OF TOKYO

UNIVERSITY OF UTAH

WASHINGTON STATE UNIVERSITY

UNIVERSITY OF WASHINGTON

$* * \quad * \quad *$
AMERICAN MATHEMATICAL SOCIETY
CHEVRON RESEARCH CORPORATION
TRW SYSTEMS
NAVAL WEAPONS CENTER




\section{Pacific Journal of Mathematics}

Vol. 30, No. $1 \quad$ September, 1969

William Wells Adams, Simultaneous diophantine approximations and cubic irrationals ..................................... 1

Heinz Bauer and Herbert Stanley Bear, Jr., The part metric in convex

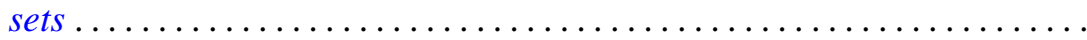

L. Carlitz, A note on exponential sums ...................... 35

Vasily Cateforis, On regular self-injective rings ................. 39

Franz Harpain and Maurice Sion, A representation theorem for measures on infinite dimensional spaces ......................... 47

Richard Earl Hodel, Sum theorems for topological spaces .............. 59

Carl Groos Jockusch, Jr. and Thomas Graham McLaughlin, Countable

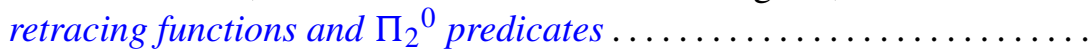

Bjarni Jónsson and George Stephen Monk, Representations of primary

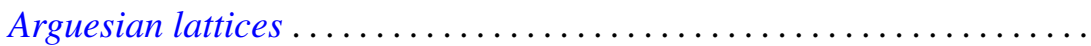

Virginia E. Walsh Knight, A continuous partial order for Peano continua...................................... 141

Kjeld Laursen, Ideal structure in generalized group algebras ........... 155

G. S. Monk, Desargues' law and the representation of primary lattices . . . 175

Hussain Sayid Nur, Singular perturbation of linear partial differential equation with constant coefficients ..........................

Richard Paul Osborne and J. L. Stern, Covering manifolds with cells ... 201

Keith Lowell Phillips and Mitchell Herbert Taibleson, Singular integrals in several variables over a local field...

James Reaves Smith, Local domains with topologically $T$-nilpotent radical....

Donald Platte Squier, Elliptic differential equations with discontinuous coefficients .................................

Tae-il Suh, Algebras formed by the Zorn vector matrix...

Earl J. Taft, Ideals in admissible algebras . .................... 259

Jun Tomiyama, On the tensor products of von Neumann algebras........ 263

David Bertram Wales, Uniqueness of the graph of a rank three group ..... 271

Charles Robert Warner and Robert James Whitley, A characterization of regular maximal ideals ......................... 